\title{
UMA AVALIAÇÃO CRÍTICA DAS FORMULAÇÕES DE FLUXO DE POTÊNCIA PARA SISTEMAS TRIFÁSICOS VIA MÉTODO DE NEWTON-RAPHSON
}

\author{
Hivy Queiroz Pereira* \\ hivyqueiroz@yahoo.com.br
}

\author{
Vander Menengoy da Costa* \\ vandermcosta@uol.com.br
}

\author{
*UFJF / Faculdade de Engenharia \\ Juiz de Fora, MG \\ Brasil
}

\section{RESUMO}

Este trabalho apresenta um estudo comparativo das características de convergência das formulações convencional polar, convencional retangular e injeção de corrente na solução do fluxo de potência trifásico. As equações não lineares referentes a cada uma das formulações são resolvidas através do método iterativo de Newton-Raphson. Sistemas trifásicos desequilibrados e mal-condicionados são testados de modo a fornecer subsídios a respeito do desempenho de cada um dos métodos. As características de convergência são avaliadas tanto em relação à variação do carregamento, quanto à variação da relação $\mathrm{R} / \mathrm{X}$ dos ramos.

PALAVRAS-CHAVE: Fluxo de Potência Trifásico, Convencional Polar, Convencional Retangular, Injeção de Corrente.

\section{ABSTRACT}

This paper presents a comparative study on convergence characteristics of the main three-phase power flow methods, namely, conventional polar, conventional rectangular and current injection formulations, for solving the threephase power flow problem. The nonlinear equations are solved iteratively through Newton-Raphson approach. Both

\footnotetext{
Artigo submetido em 14/03/2006

1a. Revisão em 05/06/2006

2a. Revisão em 15/01/2007

Aceito sob recomendação do Editor Associado

Prof. Carlos A. Castro
}

unbalanced and ill-conditioned three-phase power systems are tested in order to provide information on performance of each one of the methods under analysis. The convergence characteristics are evaluated by increasing the loading factor and the branch $\mathrm{R} / \mathrm{X}$ ratios.

KEYWORDS: Three-Phase Power Flow, Conventional Polar, Conventional Rectangular, Current Injection.

\section{NOMENCLATURA}

$n$ Número de barras do sistema;

$s$ Elemento representativo das fases $a, b$ e $c$;

$h$ Contador de iterações;

$P_{G k}^{s}+j Q_{G k}^{s}$ Potência complexa gerada na barra $k$, fase $s$;

$P_{L k}^{s}+j Q_{L k}^{s}$ Potência complexa demandada na barra $k$, fase $s$;

$P_{k}^{s}+j Q_{k}^{s}$ Potência complexa líquida injetada na barra $k$, fase $s$;

$\Delta P_{k}^{s}+j \Delta Q_{k}^{s}$ Resíduo de potência complexa líquida na barra $k$;

$V_{r k}^{s}+j V_{m k}^{s}$ Tensão complexa na barra $k$, fase $s$;

$\Delta V_{r k}^{s}+j \Delta V_{m k}^{s}$ Correção da tensão complexa na barra $k$, fase $s$;

$V_{k}^{s}$ Módulo da tensão na barra $k$, fase $s$; 
$\Delta V_{k}^{s}$ Correção do módulo da tensão na barra $k$, fase $s$;

$\theta_{k}^{s}$ Ângulo da tensão na barra $k$, fase $s$;

$\Delta \theta_{k}^{s}$ Correção do ângulo da tensão na barra $k$, fase $s$;

$I_{r k}^{s}+j I_{m k}^{s}$ Corrente elétrica injetada na barra $k$, fase $s$; $\Delta I_{r k}^{s}+j \Delta I_{m k}^{s}$ Resíduo da corrente elétrica injetada na barra $k$, fase $s$;

$\alpha_{p}$ Conjunto das fases $a, b$ e $c$;

$\Omega_{k}$ Conjunto de barras adjacentes à barra $k$, incluindo a própria barra $k$.

As matrizes serão apresentadas em negrito e os vetores em negrito sublinhado. Os subscritos esp e calc denotam valores especificados e calculados, respectivamente.

\section{INTRODUÇÃO}

As sucessivas evoluções no mercado elétrico brasileiro, a começar pela mudança do modelo de mercado de energia, tornam prementes diversos estudos de avaliação a respeito do desempenho das metodologias que hoje são usualmente utilizadas na análise da operação e do planejamento do sistema elétrico de geração, transmissão e distribuição.

Neste contexto, destaca-se primordialmente o estudo das metodologias para a solução do problema de fluxo de potência. Em linhas gerais, tal problema consiste no cálculo das tensões nas barras e dos fluxos de potência nas linhas de um sistema elétrico dado um nível de carga especificado e um programa de geração estabelecido. Tradicionalmente, o problema é abordado sob um enfoque estático, considerando-se tanto as equações algébricas de potência, expressas em coordenadas polares ou retangulares das tensões, quanto as restrições referentes aos limites das variáveis.

A análise em regime permanente dos sistemas trifásicos de transmissão é simplificada, uma vez que se supõe a operação em condições equilibradas. Por outro lado, quando o sistema de distribuição é o objeto do estudo, esta simplificação não é mais possível, uma vez que ocorre a operação de forma desbalanceada devido ao desequilíbrio entre as cargas das fases, a assimetria das linhas sem transposição, além da presença de circuitos monofásicos e bifásicos.

Ao longo dos anos, várias têm sido as contribuições no sentido de solucionar o problema do fluxo de potência em sistemas de distribuição (Luo e Semlyen, 1990; Chen et alii, 1991; Das et alii, 1994; Das et alii, 1995; Zimmerman, 1995; Garcia e Zago, 1996; Expósito e Ramos, 1999). Em Garcia et alii, 2001a é apresentada uma formulação para a solução do fluxo de potência trifásico desequilibrado em sistemas de distribuição de energia elétrica, utilizando as equações de injeção de corrente. Além disto, são ainda propostos modelos para representação das barras PV e para o controle de tensão em barras remotas. Em Garcia et alii, 2001b são apresentados os modelos matemáticos dos compensadores estáticos de reativos, reguladores de tensão e capacitores série controlados por tiristores para aplicação no fluxo de potência trifásico por injeção de corrente.

Mais recentemente, em Garcia et alii, 2004 é apresentada uma forma alternativa de representação da barra PV no método de solução do fluxo de potência trifásico via injeção de corrente. A potência reativa gerada pela barra PV é considerada como uma variável dependente, sendo os resíduos de corrente calculados em função dos resíduos de potência. Outros desenvolvimentos incluem o estudo de sistemas de distribuição trifásicos a 4 fios (Chen e Yang, 2001), a proposição de metodologia que considera as características topológicas dos sistemas de distribuição na solução do fluxo de potência (Teng, 2003) e finalmente, proposições de metodologias para solução do fluxo de potência trifásico e monofásico com otimização de passo utilizando as equações de injeção de corrente (De Oliveira e Da Costa, 2004; Ferreira e Da Costa, 2005).

Desta forma, o objetivo deste artigo é apresentar uma avaliação crítica a respeito das características de convergência das formulações convencional polar, convencional retangular e injeção de corrente na solução do fluxo de potência trifásico. As equações não lineares referentes a cada uma das metodologias são resolvidas através do método iterativo de Newton-Raphson.

\section{FLUXO DE POTÊNCIA TRIFÁSICO PO- LAR}

A formulação do fluxo de potência trifásico polar utiliza as equações de potência injetada nas barras, expressas em termos das coordenadas polares da tensão. As equações de potência ativa e reativa injetada numa barra genérica $k$, referente à fase $a$, são dadas por (1) e (2), respectivamente.

$$
\begin{aligned}
P_{k}^{a}= & {\left[\sum_{i \varepsilon \Omega_{k}} V_{k}^{a} V_{i}^{a}\left(G_{k i}^{a a} \cos \theta_{k i}^{a a}+B_{k i}^{a a} \operatorname{sen} \theta_{k i}^{a a}\right)\right]+} \\
& {\left[\sum_{i \varepsilon \Omega_{k}} V_{k}^{a} V_{i}^{b}\left(G_{k i}^{a b} \cos \theta_{k i}^{a b}+B_{k i}^{a b} \operatorname{sen} \theta_{k i}^{a b}\right)\right]+} \\
& {\left[\sum_{i \varepsilon \Omega_{k}} V_{k}^{a} V_{i}^{c}\left(G_{k i}^{a c} \cos \theta_{k i}^{a c}+B_{k i}^{a c} \operatorname{sen} \theta_{k i}^{a c}\right)\right] }
\end{aligned}
$$




$$
\begin{aligned}
Q_{k}^{a}= & {\left[\sum_{i \varepsilon \Omega_{k}} V_{k}^{a} V_{i}^{a}\left(G_{k i}^{a a} \operatorname{sen} \theta_{k i}^{a a}-B_{k i}^{a a} \cos \theta_{k i}^{a a}\right)\right]+} \\
& {\left[\sum_{i \varepsilon \Omega_{k}} V_{k}^{a} V_{i}^{b}\left(G_{k i}^{a b} \operatorname{sen} \theta_{k i}^{a b}-B_{k i}^{a b} \cos \theta_{k i}^{a b}\right)\right]+} \\
& {\left[\sum_{i \varepsilon \Omega_{k}} V_{k}^{a} V_{i}^{c}\left(G_{k i}^{a c} \operatorname{sen} \theta_{k i}^{a c}-B_{k i}^{a c} \cos \theta_{k i}^{a c}\right)\right] }
\end{aligned}
$$

A aplicação do processo iterativo de Newton-Raphson na solução das equações não lineares (1) e (2), referentes a todas as barras e a cada uma das fases, resulta no conjunto de equações (3), cuja solução representa as correções dos módulos e ângulos das tensões obtidas durante cada iteração. As equações de potência reativa nas três fases de uma barra PV são eliminadas.

$$
\left[\begin{array}{c}
\Delta \underline{\mathbf{P}}^{a b c} \\
\Delta \underline{\mathbf{Q}}^{a b c}
\end{array}\right]=\left[\begin{array}{ll}
\frac{\partial \underline{\mathbf{P}}^{a b c}}{\partial \underline{\boldsymbol{\theta}}^{a b c}} & \frac{\partial \underline{\mathbf{P}}^{a b c}}{\partial \underline{\mathbf{V}}^{a b c}} \\
\frac{\partial \underline{\mathbf{Q}}^{a b c}}{\partial \underline{\boldsymbol{\theta}}^{a b c}} & \frac{\partial \underline{\mathbf{Q}}^{a b c}}{\partial \underline{\mathbf{V}}^{a b c}}
\end{array}\right] \bullet\left[\begin{array}{c}
\Delta \underline{\boldsymbol{\theta}}^{a b c} \\
\Delta \underline{\mathbf{V}}^{a b c}
\end{array}\right]
$$

As atualizações dos módulos e ângulos das tensões são feitas de acordo com as equações (4) e (5).

$$
\begin{gathered}
\underline{\mathbf{V}}^{(h+1) s}=\underline{\mathbf{V}}^{(h) s}+\Delta \underline{\mathbf{V}}^{(h) s} \\
\underline{\boldsymbol{\theta}}^{(h+1) s}=\underline{\boldsymbol{\theta}}^{(h) s}+\Delta \underline{\boldsymbol{\theta}}^{(h) s}
\end{gathered}
$$

\section{FLUXO DE POTÊNCIA TRIFÁSICO RE- TANGULAR}

Neste caso, as equações de potência ativa e reativa expressas em termos das coordenadas retangulares da tensão, para uma barra genérica $k$, fase $a$, são dadas por:

$$
\begin{aligned}
& P_{k}^{a}=\left(V_{r k}^{a}\right)^{2} G_{k k}^{a a}+\left(V_{m k}^{a}\right)^{2} G_{k k}^{a a}+ \\
&+\sum_{\substack{i \in \Omega_{k} \\
i \neq k}}\left[V_{r k}^{a}\left(G_{k i}^{a a} V_{r i}^{a}-B_{k i}^{a a} V_{m i}^{a}\right)+\right. \\
&\left.\quad+V_{m k}^{a}\left(G_{k i}^{a a} V_{m i}^{a}+B_{k i}^{a a} V_{r i}^{a}\right)\right]+ \\
&+V_{r k}^{a} V_{r k}^{b} G_{k k}^{a b}+V_{m k}^{a} V_{m k}^{b} G_{k k}^{a b}- \\
& \quad-V_{r k}^{a} V_{m k}^{b} B_{k k}^{a b}+V_{m k}^{a} V_{r k}^{b} B_{k k}^{a b}+ \\
&+\quad \sum_{i \in \Omega_{k}}\left[V_{r k}^{a}\left(G_{k i}^{a b} V_{r i}^{b}-B_{k i}^{a b} V_{m i}^{b}\right)+\right. \\
&\left.+V_{m k}^{a}\left(G_{k i}^{a b} V_{m i}^{b}+B_{k i}^{a b} V_{r i}^{b}\right)\right]+ \\
&+V_{r k}^{a} V_{r k}^{c} G_{k k}^{a c}-V_{r k}^{a} V_{m k}^{c} B_{k k}^{a c}+ \\
&+ V_{m k}^{a} V_{m k}^{c} G_{k k}^{a c}+V_{m k}^{a} V_{r k}^{c} B_{k k}^{a c}+ \\
&+ \sum_{\substack{i \in \Omega_{k} \\
i \neq k}}\left[V_{r k}^{a}\left(G_{k i}^{a c} V_{r i}^{c}-B_{k i}^{a c} V_{m i}^{c}\right)+\right. \\
&\left.\quad+V_{m k}^{a}\left(G_{k i}^{a c} V_{m i}^{c}+B_{k i}^{a c} V_{r i}^{c}\right)\right]
\end{aligned}
$$

$$
\begin{aligned}
& Q_{k}^{a}=-\left(V_{m k}^{a}\right)^{2} B_{k k}^{a a}-\left(V_{r k}^{a}\right)^{2} B_{k k}^{a a}+ \\
&+ \sum_{\substack{i \in \Omega_{k} \\
i \neq k}}\left[V_{m k}^{a}\left(G_{k i}^{a a} V_{r i}^{a}-B_{k i}^{a a} V_{m i}^{a}\right)-\right. \\
&\left.-V_{r k}^{a}\left(G_{k i}^{a a} V_{m i}^{a}+B_{k i}^{a a} V_{r i}^{a}\right)\right]+ \\
&+ V_{m k}^{a} V_{r k}^{b} G_{k k}^{a b}-V_{m k}^{a} V_{m k}^{b} B_{k k}^{a b}- \\
&-V_{r k}^{a} V_{m k}^{b} G_{k k}^{a b}-V_{r k}^{a} V_{r k}^{b} B_{k k}^{a b}+ \\
&+ \sum_{\substack{i \in \Omega_{k} \\
i \neq k}}\left[V_{m k}^{a}\left(G_{k i}^{a b} V_{r i}^{b}-B_{k i}^{a b} V_{m i}^{b}\right)-\right. \\
&\left.-V_{r k}^{a}\left(G_{k i}^{a b} V_{m i}^{b}+B_{k i}^{a b} V_{r i}^{b}\right)\right]+ \\
&+V_{m k}^{a} V_{r k}^{c} G_{k k}^{a c}-V_{m k}^{a} V_{m k}^{c} B_{k k}^{a c}- \\
&-V_{r k}^{a} V_{m k}^{c} G_{k k}^{a c}-V_{r k}^{a} V_{r k}^{c} B_{k k}^{a c}+ \\
&+\quad \sum_{\substack{i \in \Omega_{k} \\
i \neq k}}\left[V_{m k}^{a}\left(G_{k i}^{a c} V_{r i}^{c}-B_{k i}^{a c} V_{m i}^{c}\right)-\right. \\
&\left.-V_{r k}^{a}\left(G_{k i}^{a c} V_{m i}^{c}+B_{k i}^{a c} V_{r i}^{c}\right)\right]
\end{aligned}
$$

A aplicação do processo iterativo de Newton-Raphson na solução das equações não lineares (6) e (7), referentes a todas as barras e a cada uma das fases, resulta num outro conjunto de equações semelhantes a (3), mas que cuja solução representa as correções das componentes real e imaginária das tensões. Assim sendo, a matriz Jacobiana difere da anterior, sendo calculada tomando-se as derivadas parciais das equações de potência ativa e reativa em relação a estas novas variáveis de estado.

\subsection{Tratamento das Barras PV}

Nas barras PQ os resíduos de potência ativa e reativa são conhecidos. Por outro lado, os resíduos de potência reativa não são conhecidos nas barras PV. Desta forma, torna-se necessário incluir para cada barra PV, uma equação adicional ao conjunto de equações não lineares que modelam o problema de solução do fluxo de potência. Além disto, em face da utilização das coordenadas retangulares, tal equação deverá impor a restrição ao módulo da tensão em cada barra PV. Sob este contexto, para uma barra $k$ do tipo PV, fase $s$, utiliza-se então a seguinte expressão:

$$
\left(V_{k}^{s}\right)^{2}=\left(V_{r k}^{s}\right)^{2}+\left(V_{m k}^{s}\right)^{2}
$$

Assim, a equação correspondente a ser utilizada no processo iterativo de Newton Raphson toma a seguinte forma:

$$
\left(\Delta V_{k}^{s}\right)^{2}=\frac{\partial\left(V_{k}^{s}\right)^{2}}{\partial V_{r k}^{s}} \Delta V_{r k}^{s}+\frac{\partial\left(V_{k}^{s}\right)^{2}}{\partial V_{m k}^{s}} \Delta V_{m k}^{s}
$$

Logo:

$$
\left(\Delta V_{k}^{s}\right)^{2}=2 V_{r k}^{s} \Delta V_{r k}^{s}+2 V_{m k}^{s} \Delta V_{m k}^{s}
$$

onde:

$$
\left(\Delta V_{k}^{s}\right)^{2}=\left(V_{\text {kesp }}^{s}\right)^{2}-\left(V_{k c a l c}^{s}\right)^{2}
$$




\subsection{Fluxo de Potência Trifásico Retangu- lar Convencional}

Uma vez que não se conhece a priori o valor da potência reativa gerada para as barras PV, então são eliminadas da matriz Jacobiana as linhas referentes a esta variável, inserindose nesta matriz termos diagonais elevados correspondentes às equações de potência reativa. Portanto, a variável $\Delta V_{m k}^{a b c}$ correspondente à equação eliminada fica sem efeito. De modo a contornar tal dificuldade, torna-se necessário então incluir novas linhas e colunas na matriz Jacobiana original e de linhas no vetor de resíduos.

O número de linhas e/ou colunas adicionais é o triplo do número de barras PV existentes no sistema em estudo. Conforme visto anteriormente, o objetivo da inclusão é reaver o controle sobre a componente imaginária da tensão na barra PV que foi perdido ao eliminar-se a equação de potência reativa desta barra. Assim sendo, as colunas adicionais da matriz aumentada são cópias daquelas eliminadas durante o processo de solução, enquanto que as linhas adicionais são dadas por (10). No vetor de resíduos são inseridos nas posições adicionais, os resíduos das componentes imaginárias da tensão nas barras PV. Desta forma, num sistema com $n$ barras, sendo a barra $k$ uma $\mathrm{PV}$, o sistema a ser resolvido possui a forma mostrada na equação (14). Vale ressaltar que os elementos $\frac{\partial P_{k}^{a b c}}{\partial V_{m k}^{a b c}}, \frac{\partial Q_{k}^{a b c}}{\partial V_{m k}^{a b c}}, 2 V_{r k}^{a b c}$ e $2 V_{m k}^{a b c}$ são matrizes $3 \times 3$ com a seguinte estrutura:

$$
\begin{gathered}
{\left[\begin{array}{ccc}
\frac{\partial P_{k}^{a}}{\partial V_{m k}^{a}} & \frac{\partial P_{k}^{a}}{\partial V_{m k}^{b}} & \frac{\partial P_{k}^{a}}{\partial V_{m k}^{c}} \\
\frac{\partial P_{k}^{b}}{\partial V_{m k}^{a}} & \frac{\partial P_{k}^{b}}{\partial V_{m k}^{b}} & \frac{\partial P_{k}^{b}}{\partial V_{m k}^{c}} \\
\frac{\partial P_{k}^{c}}{\partial V_{m k}^{a}} & \frac{\partial P_{k}^{c}}{\partial V_{m k}^{b}} & \frac{\partial P_{k}^{c}}{\partial V_{m k}^{c}}
\end{array}\right] ;\left[\begin{array}{ccc}
\frac{\partial Q_{k}^{a}}{\partial V_{m k}^{a}} & \frac{\partial Q_{k}^{a}}{\partial V_{m k}^{b}} & \frac{\partial Q_{k}^{a}}{\partial V_{m k}^{c}} \\
\frac{\partial Q_{k}^{b}}{\partial V_{m k}^{a}} & \frac{\partial Q_{k}^{b}}{\partial V_{m k}^{b}} & \frac{\partial Q_{k}^{b}}{\partial V_{m k}^{c}} \\
\frac{\partial Q_{k}^{c}}{\partial V_{m k}^{a}} & \frac{\partial Q_{k}^{c}}{\partial V_{m k}^{b}} & \frac{\partial Q_{k}^{c}}{\partial V_{m k}^{c}}
\end{array}\right]} \\
{\left[\begin{array}{ccc}
2 V_{r k}^{a} & 0 & 0 \\
0 & 2 V_{r k}^{b} & 0 \\
0 & 0 & 2 V_{r k}^{c}
\end{array}\right] ;\left[\begin{array}{ccc}
2 V_{m k}^{a} & 0 & 0 \\
0 & 2 V_{m k}^{b} & 0 \\
0 & 0 & 2 V_{m k}^{c}
\end{array}\right]}
\end{gathered}
$$

Após o cálculo das correções através da solução de (14), as componentes real e imaginária das tensões são atualizadas por (12) e (13).

$$
\begin{aligned}
& \underline{\mathbf{V}}_{r}^{(h+1) s}=\underline{\mathbf{V}}_{r}^{(h) s}+\Delta \underline{\mathbf{V}}_{r}^{(h) s} \\
& \underline{\mathbf{V}}_{m}^{(h+1) s}=\underline{\mathbf{V}}_{m}^{(h) s}+\Delta \underline{\mathbf{V}}_{m}^{(h) s}
\end{aligned}
$$

\subsection{Fluxo de Potência Trifásico Retangu- lar com Correção de Geração de Po- tência Reativa}

Neste processo, denotado por Retangular - Q, também ocorre a inclusão de linhas e colunas na matriz Jacobiana original e de linhas no vetor de resíduos. O número de linhas e/ou colunas adicionais é novamente o triplo do número de barras PV existentes no sistema em estudo. Contudo, o objetivo desta inclusão é agora tratar a potência reativa gerada na

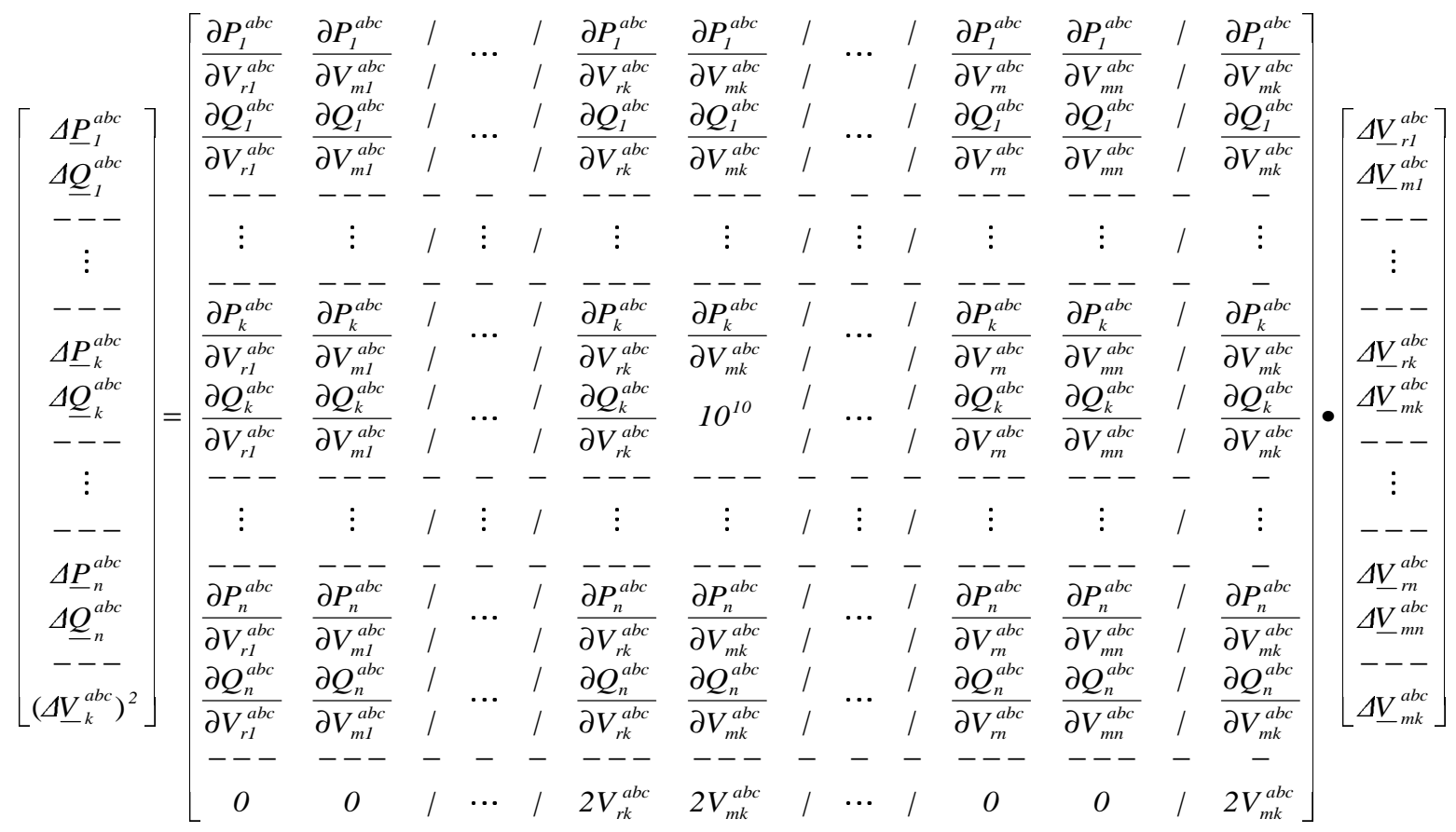




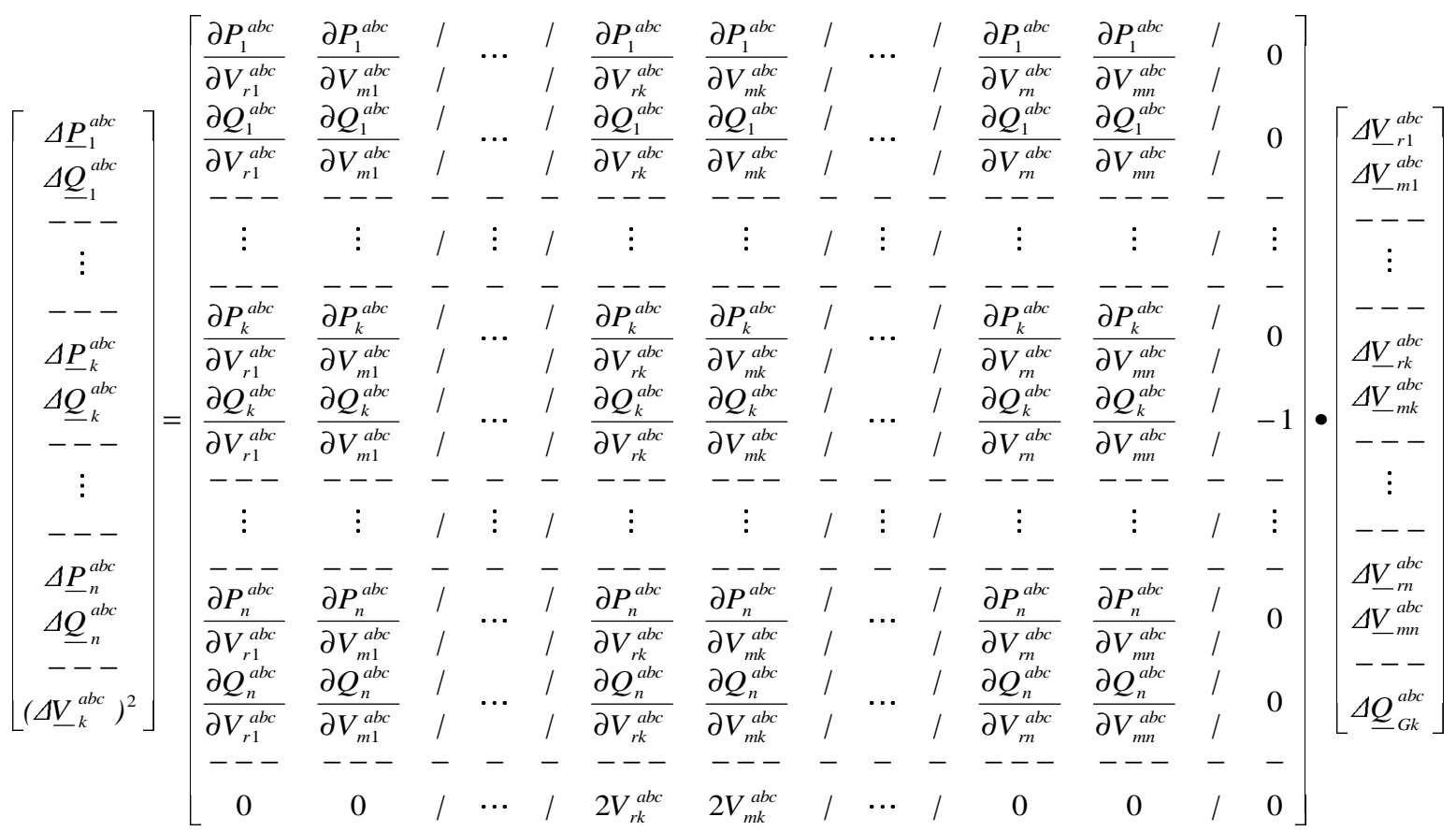

barra PV como uma variável de estado. As linhas adicionais são dadas pela equação (10), enquanto que as colunas adicionais são obtidas tomando-se as derivadas da equação (7) com relação às variáveis $Q_{G k}^{a}, Q_{G k}^{b}$ e $Q_{G k}^{c}$. Facilmente, observase que os valores destas derivadas são iguais a -1. Num sistema com $n$ barras, sendo a barra $k$ uma PV, o sistema a ser resolvido possui a forma mostrada na equação (15). O termo -1 representa uma matriz $3 \times 3$ cuja estrutura é a seguinte:

$$
\left[\begin{array}{ccc}
-1 & 0 & 0 \\
0 & -1 & 0 \\
0 & 0 & -1
\end{array}\right]
$$

$\underline{\mathbf{Q}}_{G k}^{a b c} \mathrm{e}\left(\Delta \underline{\mathbf{V}}^{a b c}\right)^{2}$ são vetores $3 \times 1$ da forma:

$$
\left[\begin{array}{l}
Q_{G k}^{a} \\
Q_{G k}^{b} \\
Q_{G k}^{c}
\end{array}\right] ;\left[\begin{array}{c}
\left(\Delta V_{k}^{a}\right)^{2} \\
\left(\Delta V_{k}^{b}\right)^{2} \\
\left(\Delta V_{k}^{c}\right)^{2}
\end{array}\right]
$$

A partir das correções calculadas por (15), os valores das componentes real e imaginária da tensão, bem como os valores de potência reativa gerada na barra $k$ são atualizados de acordo com as equações (12), (13) e (16), respectivamente. A variável $Q_{G k}^{a b c}$ é inicializada como sendo zero. Para calcular os resíduos de potência reativa são utilizados os valores atualizados de potência reativa gerada.

$$
Q_{G k}^{(h+1) s}=Q_{G k}^{(h) s}+\Delta Q_{G k}^{(h) s}
$$

\section{FLUXO DE POTÊNCIA TRIFÁSICO VIA INJEÇÃO DE CORRENTE}

Enquanto as metodologias apresentadas nas seções 2 e 3 utilizam expressões referentes às potências ativa e reativa injetadas em cada barra, o método via injeção de corrente utiliza, como a própria nomenclatura indica, expressões relativas a correntes. Esta formulação foi inicialmente proposta em Da Costa et alii, 1999 para sistemas monofásicos, sendo estendida para sistemas trifásicos em Garcia et alii, 2000.

As equações de corrente a serem resolvidas no problema de fluxo de potência referentes a uma barra genérica $k$, fase $s$, separadas em termos de suas componentes real e imaginária, denotadas por I's e I's ${ }_{\mathrm{mk}}$ respectivamente, são dadas por Garcia et alii, 2000:

$$
\begin{aligned}
& \sum_{i \in \Omega_{k}} \sum_{t \in \alpha_{p}}\left(G_{k i}^{s t} V_{r i}^{t}-B_{k i}^{s t} V_{m i}^{t}\right)-\frac{P_{k}^{s} V_{r k}^{s}+Q_{k}^{s} V_{m k}^{s}}{\left(V_{r k}^{s}\right)^{2}+\left(V_{m k}^{s}\right)^{2}}=0 \\
& \sum_{i \in \Omega_{k}} \sum_{t \in \alpha_{p}}\left(G_{k i}^{s t} V_{m i}^{t}+B_{k i}^{s t} V_{r i}^{t}\right)-\frac{P_{k}^{s} V_{m k}^{s}-Q_{k}^{s} V_{r k}^{s}}{\left(V_{r k}^{s}\right)^{2}+\left(V_{m k}^{s}\right)^{2}}=0
\end{aligned}
$$

Para cada barra PV são incluídas 3 linhas e 3 colunas adicionais na matriz Jacobiana primitiva. A equação adotada para a imposição da restrição da tensão numa barra $k$ do tipo PV é a mesma descrita por (10). Assim, num sistema com $n$ barras, sendo a barra $k$ uma PV, a aplicação do método de Newton- 
Raphson para solução de (17) e (18) resulta no conjunto de equações descrito por (23).

Os resíduos de corrente em (23) para uma barra $k$ e fase $s$ são obtidos através das equações (19) e (20):

$$
\begin{aligned}
& \Delta I_{m k}^{s}= \frac{1}{\left(V_{k}^{s}\right)^{2}}\left(\left(P_{G k}^{s}-P_{L k}^{s}\right)\left(V_{m k}^{s}\right)-\right. \\
&\left.-\left(Q_{G k}^{k}-Q_{L k}^{s}\right)\left(V_{r k}^{s}\right)-\left(V_{k}^{s}\right)^{2}\left(I_{m k}^{s}\right)\right) \\
& \Delta I_{r k}^{s}= \frac{1}{\left(V_{k}^{s}\right)^{2}}\left(\left(P_{G k}^{s}-P_{L k}^{s}\right)\left(V_{r k}^{s}\right)+\right. \\
&\left.\quad+\left(Q_{G k}^{s}-Q_{L k}^{s}\right)\left(V_{m k}^{s}\right)-\left(V_{k}^{s}\right)^{2}\left(I_{r k}^{s}\right)\right)
\end{aligned}
$$

Para as barras PQ, as variáveis $P_{G}$ e $Q_{G}$ são iguais a zero em (19) e (20). De (23) observa-se que os elementos fora da diagonal da matriz Jacobiana são idênticos aos correspondentes elementos da matriz admitância nodal. Por outro lado, os elementos diagonais dependem do modelo de carga adotado. Neste estudo, as cargas são consideradas do tipo potência constante. Obviamente, cada um destes elementos será um bloco de ordem (6x6). As derivadas que surgem nas colunas adicionais são dadas por:

$$
\begin{gathered}
\frac{\partial I_{m k}^{\prime} s}{\partial Q_{k}^{s}}=\frac{V_{r k}^{s}}{\left(V_{k}^{s}\right)^{2}} \\
\frac{\partial I_{r k}^{\prime} s}{\partial Q_{k}^{s}}=-\frac{V_{m k}^{s}}{\left(V_{k}^{s}\right)^{2}}
\end{gathered}
$$

Após o cálculo das correções através da solução do sistema (23), as componentes real e imaginária das tensões são atualizadas por (12) e (13). Vale ressaltar que no caso do cálculo dos resíduos de corrente das barras PV a variável $Q_{G k}^{s}$ é atualizada a cada iteração através de (16).

\section{ALGORITMO DE SOLUÇÃO}

O algoritmo para a solução do fluxo de potência em qualquer das metodologias descritas é composto pelas seguintes etapas:

- Passo 1: Determina-se a matriz admitância nodal trifásica $\mathbf{Y}^{a b c}$;

- Passo 2: Calculam-se os resíduos de potência ativa e reativa de todas as barras, através de (24) a (27) para as metodologias polar e retangulares. Por outro lado, para a metodologia injeção de corrente, calculam-se os resíduos de corrente através de (19) e (20).

$$
\begin{gathered}
\underline{\mathbf{I}}^{a b c}=\mathbf{Y}^{a b c} \cdot \underline{\mathbf{V}}^{a b c} \\
\underline{\mathbf{P}}_{c a l c}^{a b c}+j \underline{\mathbf{Q}}_{c a l c}^{a b c}=\underline{\mathbf{V}}^{a b c} \cdot\left(\underline{\mathbf{I}}^{a b c}\right)^{*} \\
\Delta \underline{\mathbf{P}}^{a b c}=\underline{\mathbf{P}}^{a b c}-\underline{\mathbf{P}}_{c a l c}^{a b c} \\
\Delta \underline{\mathbf{Q}}^{a b c}=\underline{\mathbf{Q}}^{a b c}-\underline{\mathbf{Q}}_{c a l c}^{a b c}
\end{gathered}
$$

- Passo 3: Comparam-se os resíduos com uma tolerância pré-definida. No caso específico deste trabalho igual a $10^{-5} \mathrm{pu}$. Se o resíduo máximo for menor que esta tolerância, o processo é finalizado. Caso o resíduo máximo seja maior que esta tolerância, então as correções são calculadas. De acordo com a metodologia escolhida, o sistema de equações a ser resolvido será dado por (3), (14), (15) ou (23).

- Passo 4: A atualização das variáveis de estado também depende do método escolhido, sendo feita de acordo com as equações (4) e (5) ou (12) e (13) ou (12), (13) e (16).

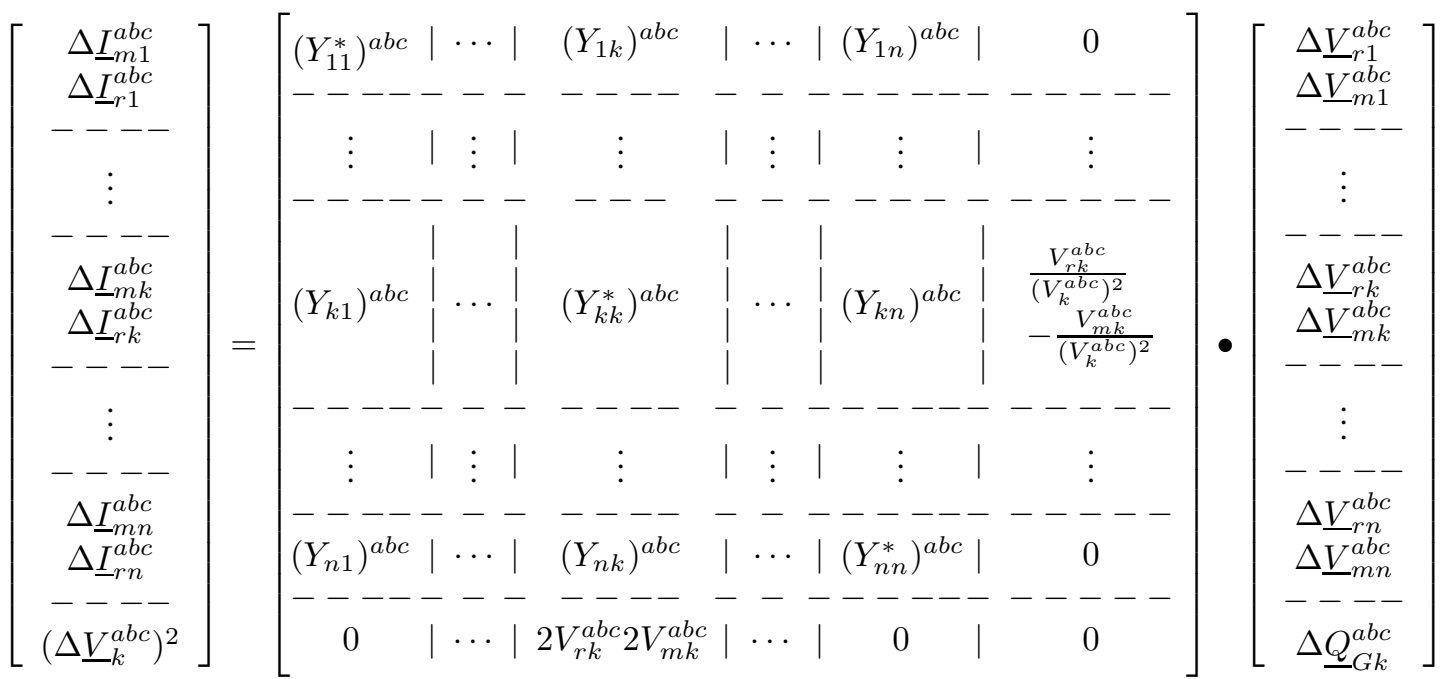




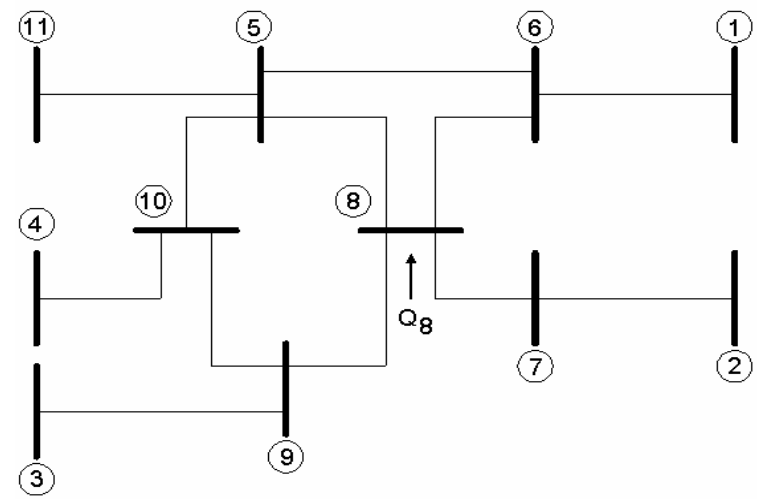

Figura 1: Topologia do Sistema Mal-Condicionado

- Passo 5: Com os novos valores de tensão, os resíduos são recalculados em todas as barras. Se estes ainda forem maiores que a tolerância, uma nova matriz Jacobiana é calculada a partir dos valores atualizados. Este passo se repete até que os resíduos encontrados sejam menores que a tolerância estipulada no passo 3 .

\section{RESULTADOS}

Os resultados apresentados referem-se a dois sistemas testes. A rede mal-condicionada de 11 barras (Castro e Braz, 1997) apresentada na Figura 1 é um sistema de transmissão, sendo convenientemente adaptado para as necessidades deste trabalho, isto é, os valores de potência ativa e reativa da fase $a$ foram repetidos para as fases $b$ e $c$ e os valores de tensão da fase ativeram seus módulos mantidos e suas fases defasadas em -120 e 120 graus, para as fases $b$ e $c$, respectivamente. O sistema $\mathrm{C} 37 \mathrm{pv}$ é uma adaptação de um alimentador real de 37 barras cuja topologia está mostrada na Figura 2. Esta adaptação refere-se ao fato de se considerar a barra 25 como sendo PV, com o módulo da tensão especificado em 1 p.u. e a potência ativa gerada nula para as três fases.

Nas figuras e tabelas desta seção, a legenda retangular significa os resultados gerados por ambas as metodologias em coordenadas retangulares apresentadas neste artigo. As legendas retangular e retangular-Q em conjunto serão utilizadas quando tais metodologias apresentarem trajetórias de convergência diferentes.

A simulação dos resultados é feita variando-se gradativamente a relação $\mathrm{R} / \mathrm{X}$ dos ramos e o carregamento dos sistemas. Os aumentos gradativos são feitos até que o processo iterativo não mais convirja, ou seja, quando o resíduo máximo ao final de cada iteração é maior que a tolerância prédefinida para um máximo de 50 iterações. Para o sistema de 11 barras são considerados diferentes valores de potência reativa injetada na barra $8\left(Q_{8}\right)$.

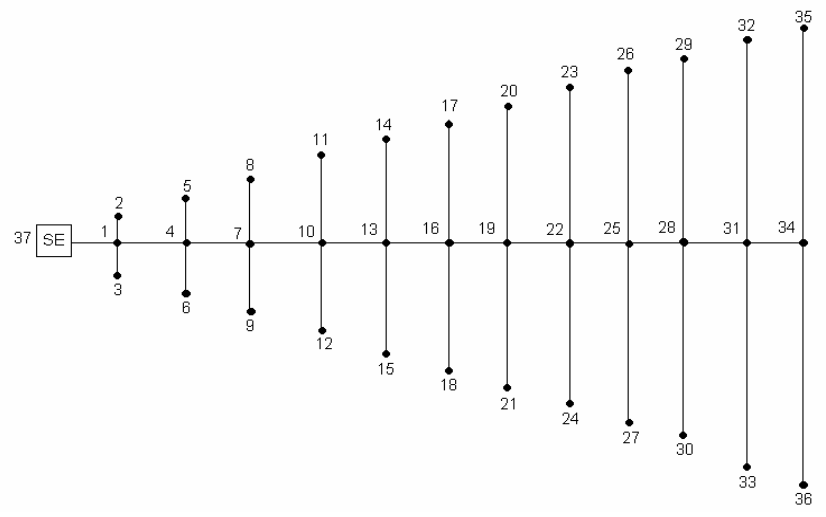

Figura 2: Topologia do Sistema C37pv

\subsection{Sistema Mal-Condicionado Caso Base}

A rede de 11 barras é mal-condicionada, sendo a solução do problema de fluxo de potência fortemente dependente do valor da injeção de potência reativa na barra 8 (Castro e Braz, 1997). Basicamente, quatro regiões são definidas em função desta injeção, notadamente, $\mathrm{Q}_{8} \geq 123 \mathrm{MVar}, 120<\mathrm{Q}_{8}$ $<123$ MVar, $101<\mathrm{Q}_{8} \leq 120$ MVar e $\mathrm{Q}_{8} \leq 101 \mathrm{MVar}$. Em cada uma destas regiões, o fluxo de potência apresenta características específicas não somente com relação a sua convergência, mas também no que diz respeito ao perfil de tensão resultante. Assim sendo, esta seção apresenta resultados para quatro valores de $\mathrm{Q}_{8}$, um em cada região anteriormente descrita.

As Figuras 3 a 6 apresentam os perfis de tensão correspondentes aos valores de $\mathrm{Q}_{8}$ iguais a 123, 122, 120 e 101 MVar, respectivamente. Para $\mathrm{Q}_{8}=123$ MVar, Figura 3, o método de solução polar converge em 11 iterações com módulos de tensão altos nas barras de carga, variando de 1,33 a 1,44 pu. Estes mesmos valores de tensão são obtidos em 6 iterações utilizando-se as formulações retangulares e de injeção de corrente. Para $\mathrm{Q}_{8}=122$ MVar, Figura 4, o fluxo de potência polar converge em 15 iterações com módulos de tensão baixos nas barras de carga, variando de 0,88 a $0,93 \mathrm{pu}$. Por outro lado, as formulações retangulares e de injeção de corrente convergem em 6 iterações para módulos de tensão altos variando de 1,32 a 1,43 pu.Para $\mathrm{Q}_{8}=120$ MVar, Figura 5, o método de solução polar converge em 10 iterações para módulos de tensão baixos nas barras de carga, variando de 0,87 a 0,93 pu. Por outro lado, as formulações retangulares e de injeção de corrente convergem em 6 iterações para módulos de tensão altos, variando de 1,32 a 1,43 pu. Finalmente, para $\mathrm{Q}_{8}=101$ MVar o fluxo de potência polar não converge. As formulações retangulares e de injeção de corrente convergem em 6 e 7 iterações, respectivamente, para os valores de tensão 


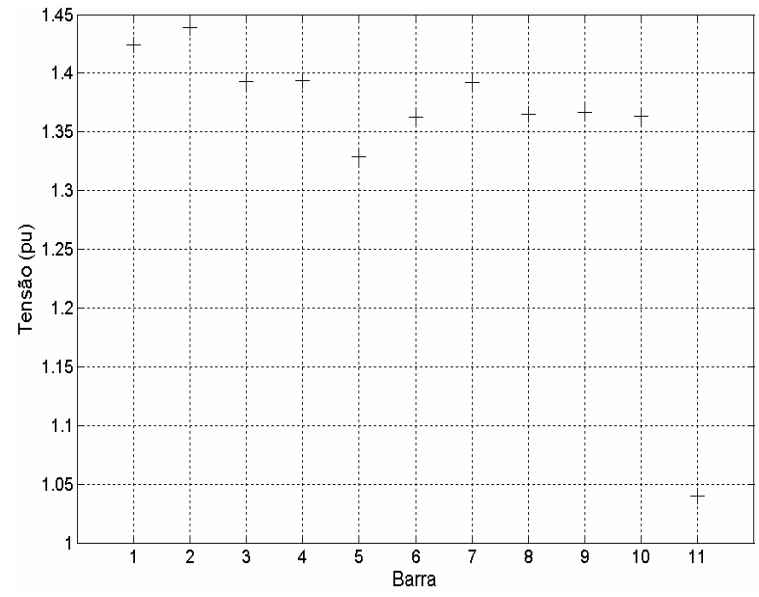

Figura 3: Perfil de Tensão Sistema 11 barras $-\mathrm{Q}_{8}=123 \mathrm{MVar}$

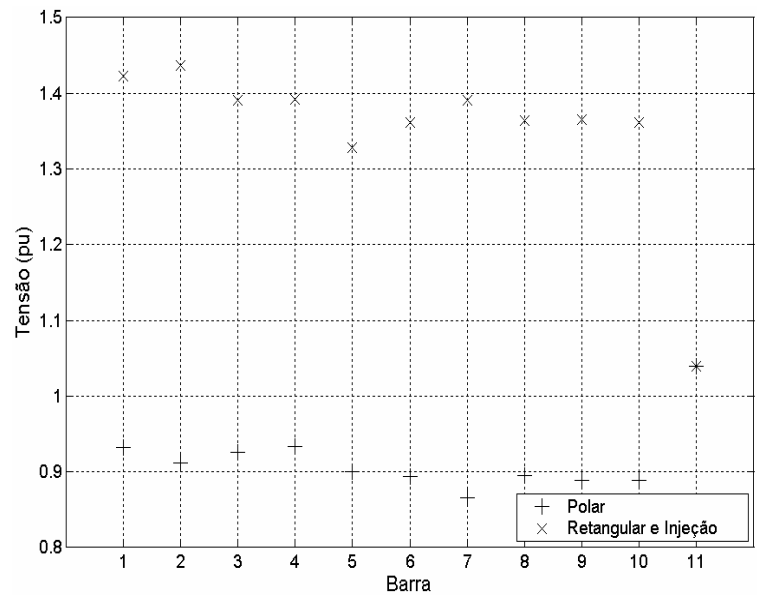

Figura 4: Perfil de Tensão Sistema 11 barras $-\mathrm{Q}_{8}=122 \mathrm{MVar}$

mostrados na Figura 6.

\subsection{Sistemas Equilibrados}

As Figuras 7 e 8 mostram o número de iterações referentes a cada um dos sistemas, quando a relação $\mathrm{R} / \mathrm{X}$ é aumentada. Por outro lado, as Figuras 9 e 10 mostram o número de iterações à medida que o carregamento é aumentado. $\mathrm{O}$ valor de Q8 considerado nestas simulações é 120 MVar.

Das Figuras 7 e 9 observa-se que para o sistema malcondicionado de 11 barras, o método polar consome mais iterações que as metodologias retangulares e a formulação via injeção de corrente. Com relação às Figuras 8 e 10 observase que para o sistema bem-condicionado de 37 barras, todas as metodologias apresentadas neste trabalho possuem um desempenho semelhante, isto é, convergem na maioria dos casos, com o mesmo número de iterações, diferindo obvia-

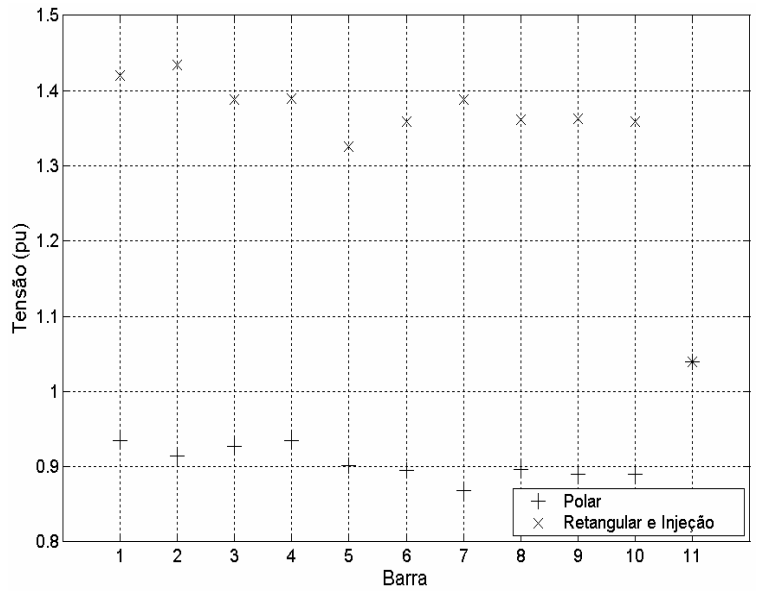

Figura 5: Perfil de Tensão Sistema 11 barras $-\mathrm{Q}_{8}=120 \mathrm{MVar}$

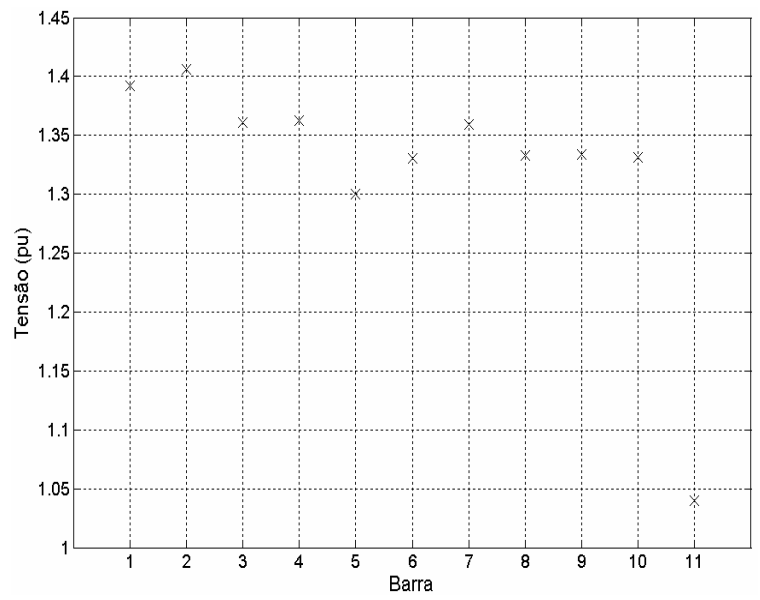

Figura 6: Perfil de Tensão Sistema 11 barras $-\mathrm{Q}_{8}=101 \mathrm{MVar}$

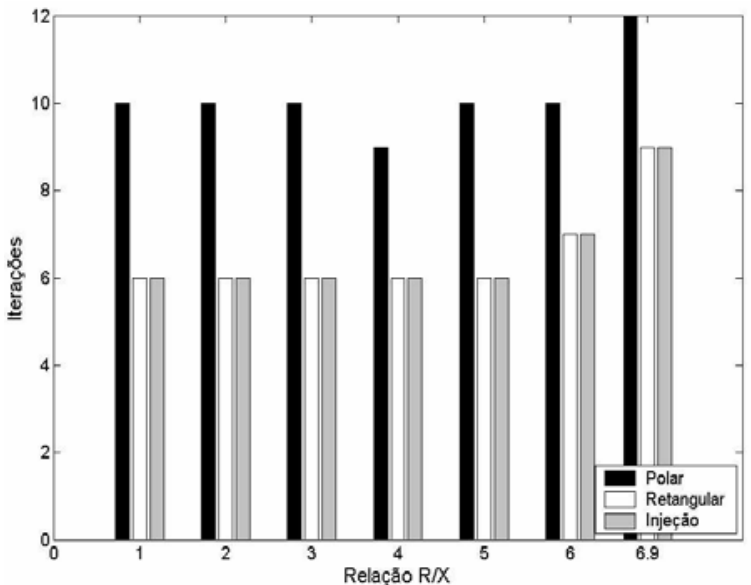

Figura 7: Sistema 11 Barras $-Q_{8}=120 \mathrm{MVar}$ 


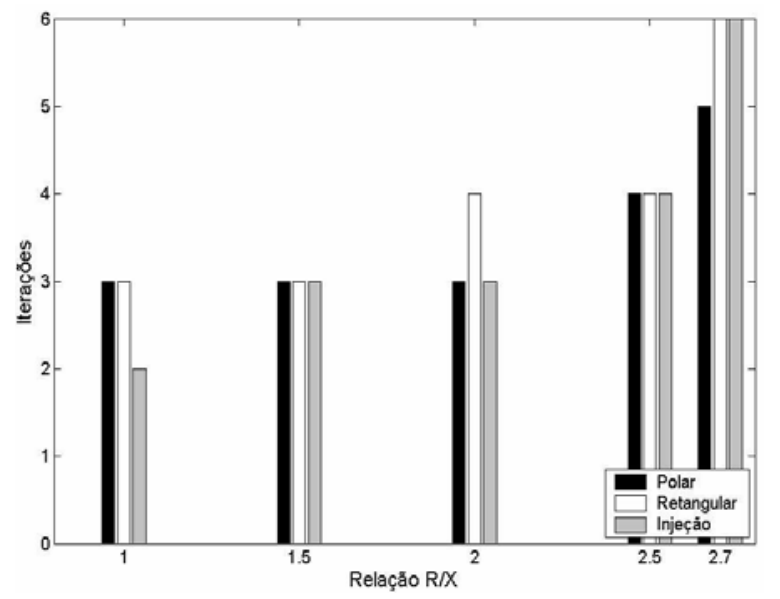

Figura 8: Sistema C37pv

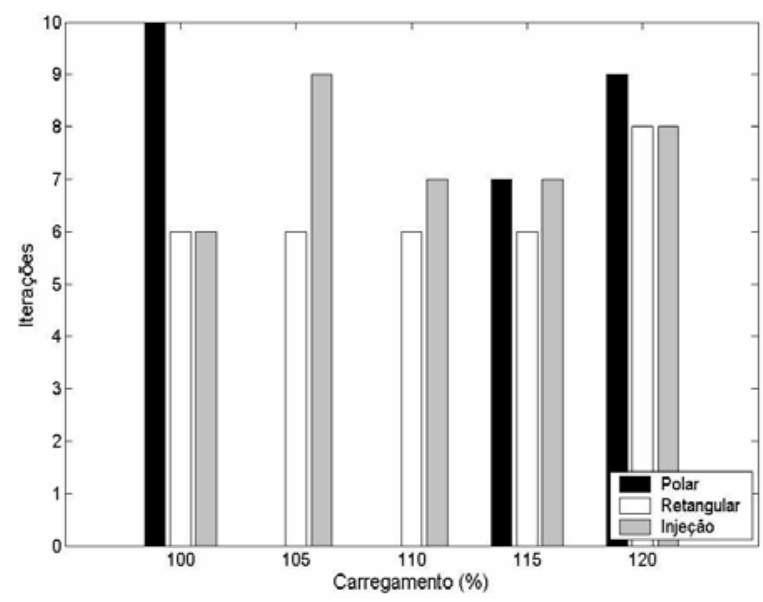

Figura 9: Sistema 11 Barras $-Q_{8}=120 \mathrm{MVAr}$

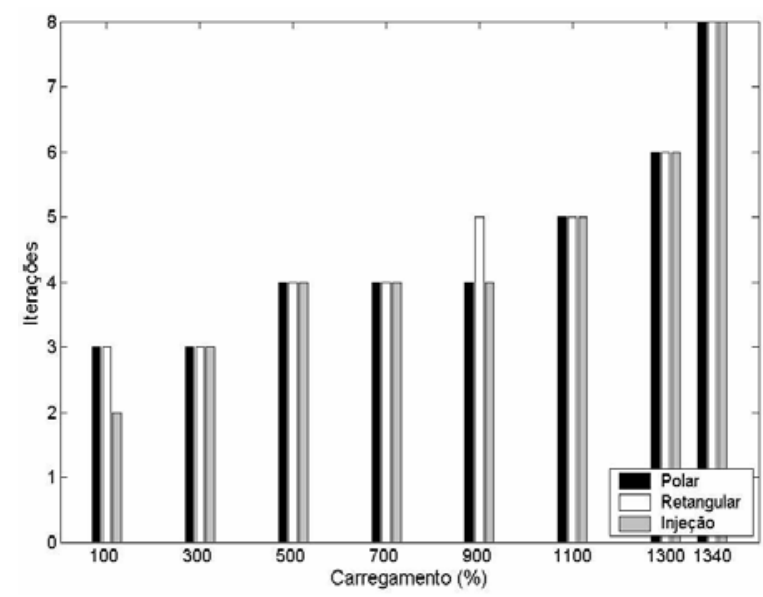

Figura 10: Variação no Carregamento - Sistema C37pv

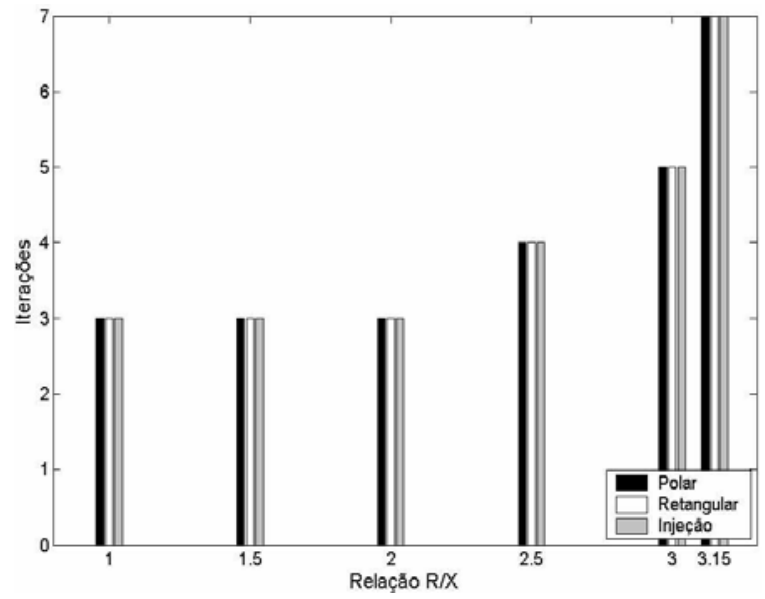

Figura 11: Variação na Relação R/X - Sistema Equilibrado -3 Barras PV

mente na trajetória de convergência, mas atingindo a mesma solução.

A formulação polar não converge para os carregamentos de 105\% e 110\%, conforme mostrado na Figura 9. Para o carregamento de $100 \%$ a formulação polar converge para módulos de tensão na faixa de 0,9 pu, enquanto que as formulações retangulares e de injeção de corrente convergem para módulos de tensão na faixa de 1,3 pu. Para o carregamento de 105\% a formulação injeção de corrente converge para módulos de tensão na faixa de $0,9 \mathrm{pu}$, enquanto que os retangulares convergem para tensões na faixa de 1,3 pu. Por outro lado, ambas as formulações convergem para tensões na faixa de 1,3 pu para o carregamento de $110 \%$. O fato da formulação polar quando converge, resultar soluções diferentes daquelas obtidas pelos outros três métodos para todos os valores de carregamento testados, pode ser a razão pela qual esta formulação não ter convergido para os dois valores anteriormente citados.

As Figuras 11 e 12 apresentam resultados referentes ao sistema de 37 barras, no qual são inseridas mais duas barras de geração adicionais. O objetivo é verificar a robustez das metodologias na presença de um maior número de barras PV. Tal fato, inclusive, pode caracterizar uma situação real de redes de distribuição com mais de um regulador de tensão. Desta forma, além da barra 25 previamente definida como barra de geração, são também escolhidas as barras 7 e 16, ambas com tensão fixada em 1 pu e potência ativa gerada nula nas três fases. A Figura 11 mostra que as metodologias apresentam desempenhos idênticos quanto à variação da relação $\mathrm{R} / \mathrm{X}$. Com relação ao aumento do carregamento, observa-se da Figura 12 que a metodologia polar apresenta de uma forma geral um desempenho superior, especialmente em situações de alto carregamento. 


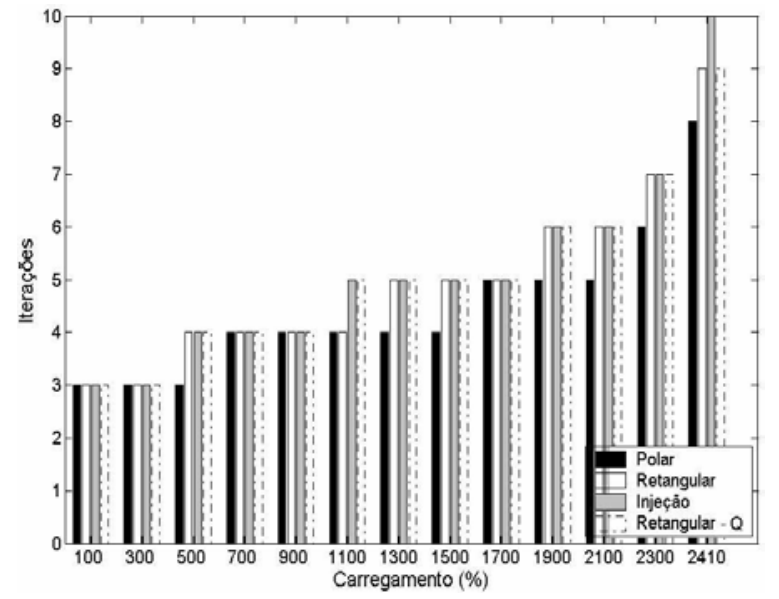

Figura 12: Variação no Carregamento - Sistema Equilibrado - 3 Barras PV

Tabela 1: Desequilíbrios no Sistema 11 barras

\begin{tabular}{|c|c|c|c|c|c|c|}
\hline Barra & $\begin{array}{c}P_{L a} \\
(p u)\end{array}$ & $\begin{array}{c}\mathrm{P}_{L b} \\
(p u)\end{array}$ & $\begin{array}{c}P_{L c} \\
(p u)\end{array}$ & $\begin{array}{c}Q_{L a} \\
(p u)\end{array}$ & $\begin{array}{c}Q_{L b} \\
(p u)\end{array}$ & $\begin{array}{c}Q_{L c} \\
(p u)\end{array}$ \\
\hline 3 & 0,75 & 0 & 0,75 & $-0,42$ & 0 & $-0,42$ \\
\hline 11 & 2 & 3 & 1 & 0 & 0,50 & 0 \\
\hline
\end{tabular}

Tabela 2: Desequilíbrios no Sistema C37pv

\begin{tabular}{|c|c|c|c|c|c|c|}
\hline Barra & $\begin{array}{c}P_{L a} \\
(p u)\end{array}$ & $\begin{array}{c}P_{L b} \\
(p u)\end{array}$ & $\begin{array}{c}P_{l c} \\
(p u)\end{array}$ & $\begin{array}{c}Q_{L a} \\
(p u)\end{array}$ & $\begin{array}{c}Q_{L b} \\
(p u)\end{array}$ & $\begin{array}{c}Q_{L c} \\
(p u)\end{array}$ \\
\hline 5 & 0,30 & 0,20 & 0,30 & 0,17 & 0 & 0,17 \\
\hline 15 & 0,80 & 0,75 & 0,90 & 0,43 & 0,43 & 0,43 \\
\hline 36 & 1,81 & 1,81 & 1,81 & 0 & 0 & 0 \\
\hline
\end{tabular}

\subsection{Sistemas Desequilibrados}

Para validar as metodologias frente a sistemas desequilibrados, são efetuadas modificações aleatórias na potência demandada de algumas barras dos sistemas em estudo. As Tabelas 1 e 2 apresentam os desequilíbrios propostos para os sistemas 11 barras e C37pv, respectivamente. $\mathrm{O}$ valor de $\mathrm{Q}_{8}$ considerado nestas simulações é 101 MVar.

As Figuras 13 e 14 mostram o número de iterações quando a relação R/X é aumentada. Da mesma forma, as Figuras 15 e 16 mostram o número de iterações à medida que o carregamento é aumentado. Das Figuras 13 e 15 observa-se que para o sistema mal-condicionado desequilibrado, o método polar não converge em alguns casos, enquanto que as metodologias retangulares e de injeção de corrente convergem. Para o sistema bem-condicionado desequilibrado, conforme mostrado nas Figuras 14 e 16, todas as metodologias apre-

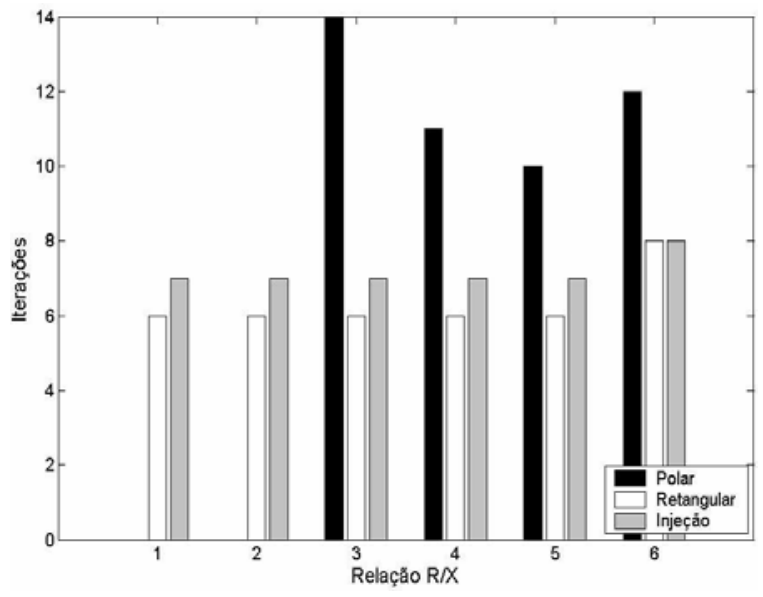

Figura 13: Sistema 11 Barras Desequilibrado $Q_{8}=101$ MVAr

sentam desempenhos semelhantes, convergindo basicamente no mesmo número de iterações e atingindo a mesma solução em todos os níveis de carregamento e variações da relação $\mathrm{R} / \mathrm{X}$.

Para todos os valores da relação R/X mostrados na Figura 13 , as formulações retangulares e de injeção de corrente convergem para a mesma solução, caracterizada pelo módulo de tensão acima de 1,1 pu. Para os valores desta relação iguais a 3 e 4, a formulação polar consome 14 e 11 iterações, respectivamente, convergindo para uma solução caracterizada pelo módulo de tensão menor que 1 pu. Por outro lado, para as relações iguais a 5 e 6 , a formulação polar converge para a mesma solução obtida pelas formulações retangulares e de injeção de corrente. É possível constatar que a formulação polar converge para tensões baixas quando as menores relações R/X são consideradas. Assim sendo, para esta situação específica é possível relacionar o desempenho da metodologia polar ao valor da relação $\mathrm{R} / \mathrm{X}$, ou seja, como os valores de tensão diminuem à medida que a relação $\mathrm{R} / \mathrm{X}$ decresce, então é possível concluir que a formulação polar gaste mais iterações para a convergência do processo iterativo em relações $\mathrm{R} / \mathrm{X}$ mais baixas, podendo até não convergir.

As Figuras 17 a 22 mostram o perfil de tensão correspondente a $100 \%$ e $1340 \%$ de carregamento mostrado na Figura 16 , considerando-se o sistema C37pv desequilibrado. Conforme esperado, o perfil de tensão referente ao carregamento maior é caracterizado por módulos de tensão mais baixos. Além disto, em todas estas figuras é possível observar que a tensão na barra de geração 25 é mantida constante em 1 pu, conforme previamente estabelecido.

Para o sistema de 37 barras desequilibrado e com as três barras de geração definidas na seção 6.2 , observa-se que todas 


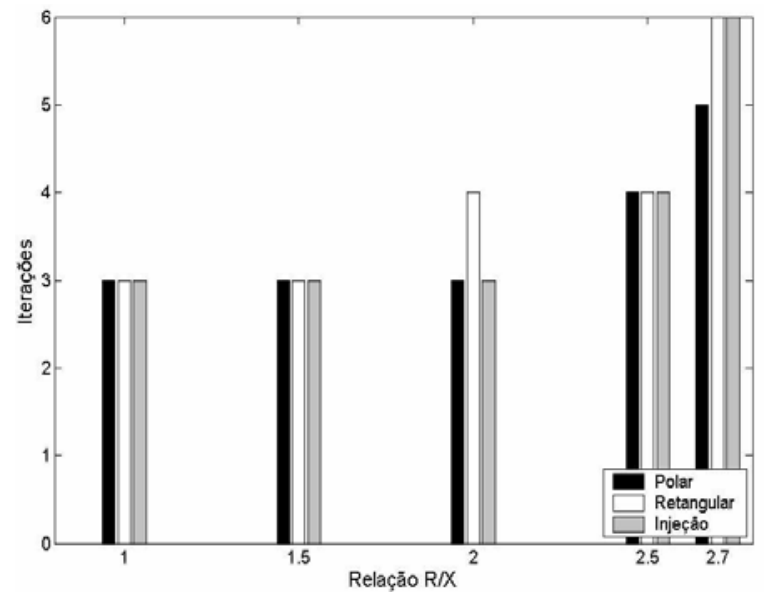

Figura 14: Sistema C37pv Desequilibrado

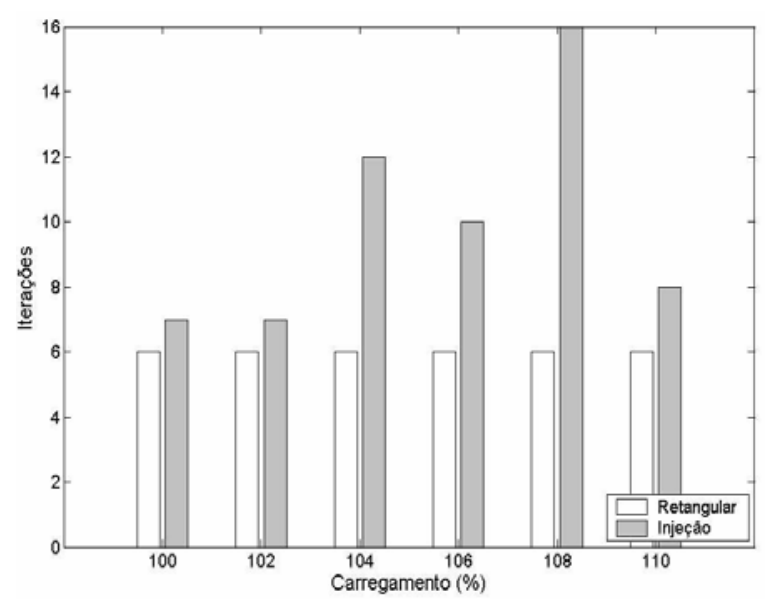

Figura 15: Sistema 11 Barras Desequilibrado $-Q_{8}=101$ MVar

as metodologias convergem exatamente no mesmo número de iterações mostrado nas Figuras 11 e 12. A única exceção refere-se ao carregamento de $2410 \%$, onde são necessárias 7, 8, 8 e 8 iterações considerando-se as formulações polar, retangular, retangular-Q e injeção de corrente, respectivamente.

\subsection{Soluções Múltiplas}

Nem sempre as metodologias aplicadas a um mesmo caso, convergem para o mesmo ponto de solução em se tratando de sistemas mal-condicionados. A veracidade desta situação peculiar foi comprovada fazendo a solução encontrada pelos métodos retangulares e injeção de corrente como dados de entrada para a formulação polar e também os resultados da formulação polar como dados de entrada para as outras três metodologias. Nestes casos, observa-se que os algoritmos

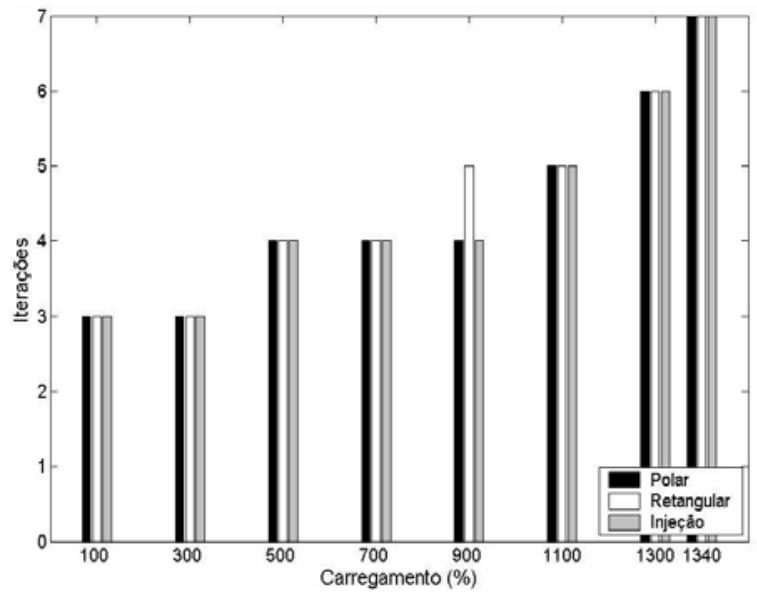

Figura 16: Sistema C37pv Desequilibrado

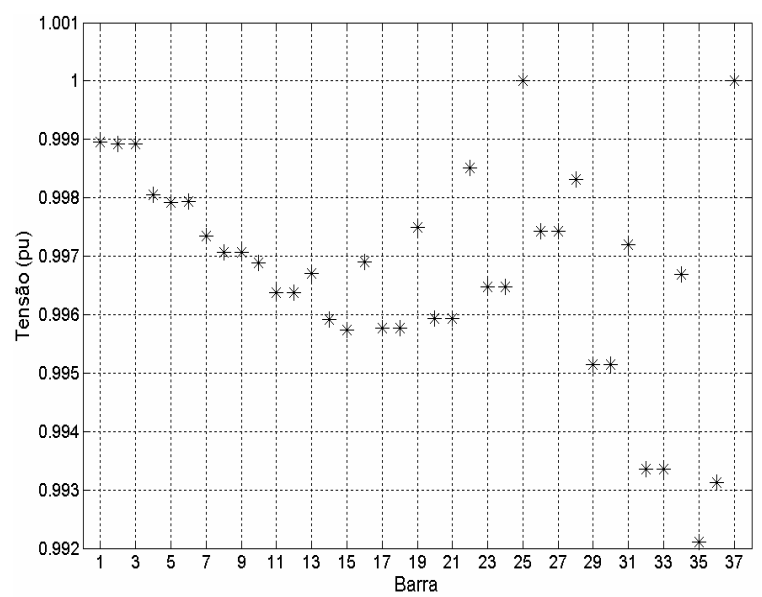

Figura 17: Perfil de Tensão para 100\% - Fase a

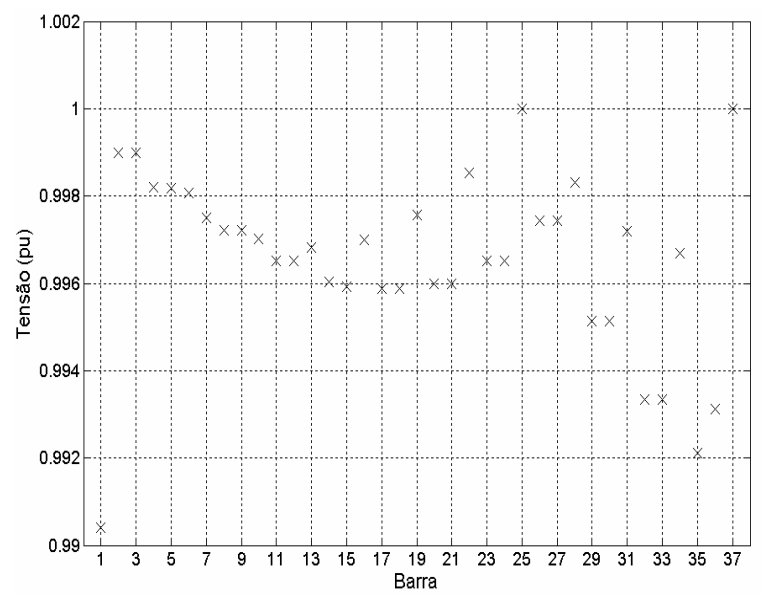

Figura 18: Perfil de Tensão para 100\% - Fase b 


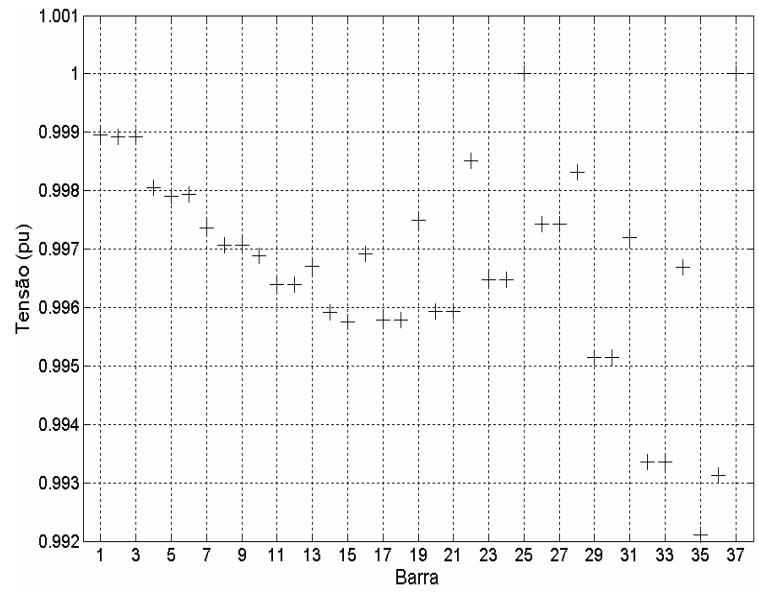

Figura 19: Perfil de Tensão para 100\% - Fase c

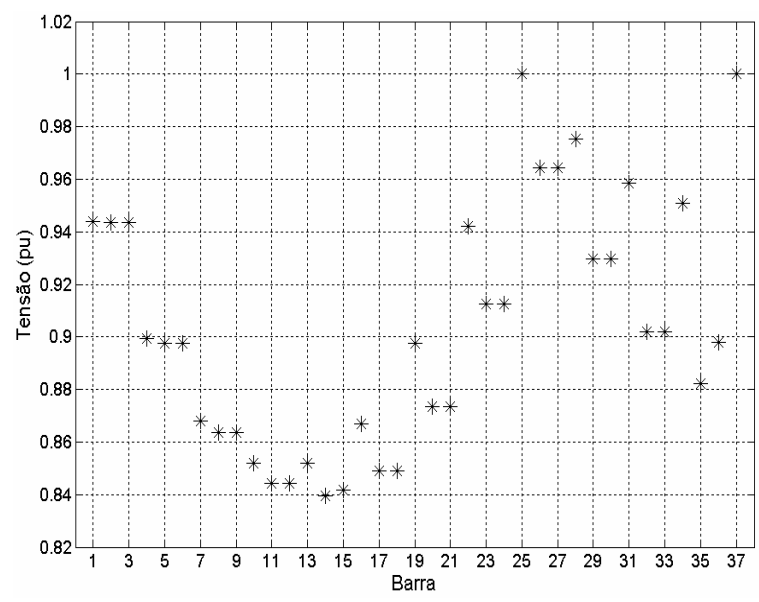

Figura 20: Perfil de Tensão para 1340\% - Fase a

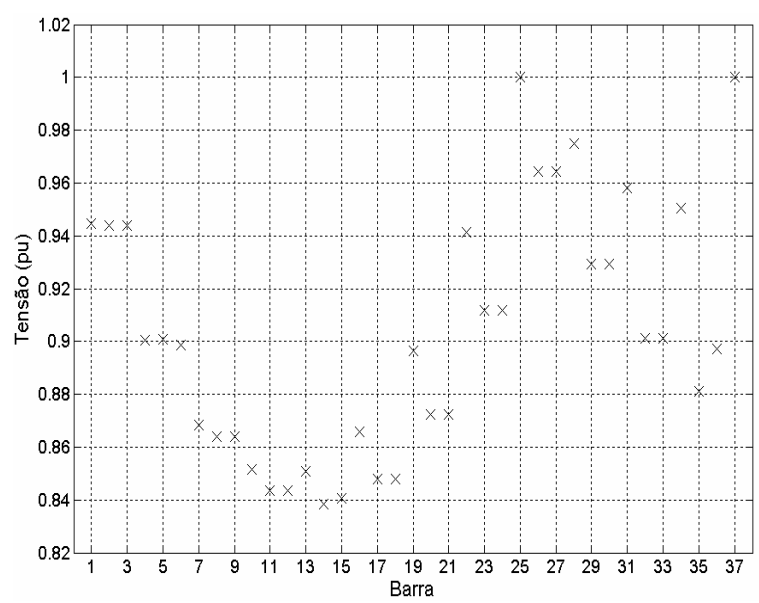

Figura 21: Perfil de Tensão para 1340\% - Fase b

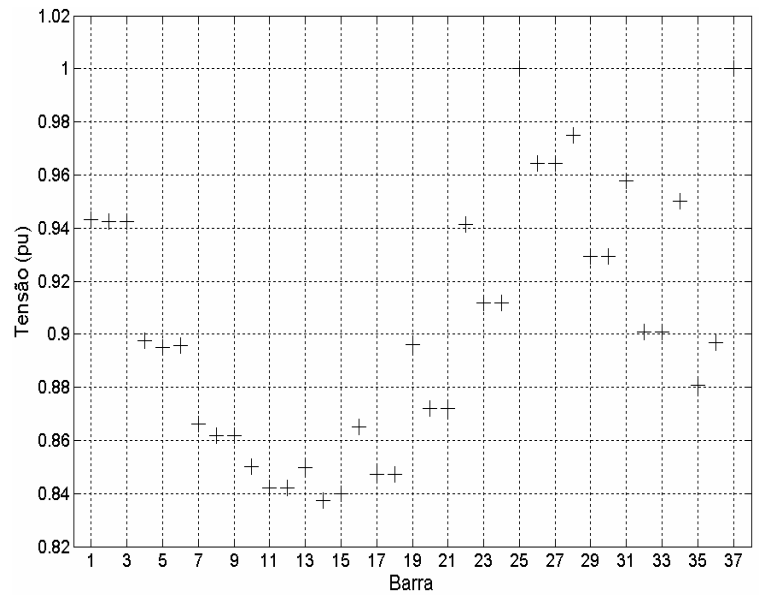

Figura 22: Perfil de Tensão para 1340\% - Fase c

não entram no processo iterativo, ou em outras palavras, os dados de entrada realmente correspondem a uma solução do sistema.

Neste contexto, o sistema 11 barras, equilibrado ou não, com as variações de $Q_{8}$, do carregamento e da relação $\mathrm{R} / \mathrm{X}$ apresentou resultados muito interessantes, conforme mostrados na Tabela 3. A última coluna indica os valores mínimo e máximo de tensão no ponto de solução. O valor 1,04 é a tensão na barra de referência, para a qual a tabela também exibe os valores de potência ativa e reativa geradas. Os demais casos não citados referentes ao sistema 11 barras, quando há convergência, caminham para a mesma solução.

Os resultados para $Q_{8}=120 \mathrm{MVar}$, referentes ao caso base, coincidem com aqueles apresentados em Castro e Braz, 1997.

\section{CONCLUSÕES}

Este trabalho propõe uma avaliação crítica a respeito do desempenho das formulações de fluxo de potência na solução de sistemas trifásicos através do método iterativo de NewtonRaphson. As metodologias convencional polar, retangular convencional, retangular com correção da geração de potência reativa e via equações de injeção de corrente são convenientemente analisadas.

Para sistemas bem-condicionados, equilibrados ou não, todas as metodologias descritas apresentam desempenho semelhante, isto é, convergem basicamente com o mesmo número de iterações e atingem o mesmo ponto de solução nos diversos níveis de carregamento e variação da relação R/X. Desta forma, para esta situação em particular, qualquer uma das metodologias estudadas pode a princípio ser utilizada na solução do problema de fluxo de potência trifásico. 
Tabela 3: Soluções Múltiplas do Sistema 11 Barras

\begin{tabular}{|c|c|c|c|c|c|c|}
\hline $\begin{array}{c}\mathrm{Q}_{8} \\
\text { (MVAr) }\end{array}$ & $\mathrm{R} / \mathrm{X}$ & $\begin{array}{l}\text { Carrega } \\
\text { mento }\end{array}$ & Método & $\begin{array}{l}\mathrm{P}_{\mathrm{G}}^{\mathrm{a}} \\
\text { (p.u.) }\end{array}$ & $\begin{array}{c}\mathrm{Q}_{\mathrm{G}}^{\mathrm{a}} \\
\text { (p.u.) }\end{array}$ & $\begin{array}{l}\text { Intervalo } \\
\text { de } \\
\text { Tensões } \\
\text { (p.u.) }\end{array}$ \\
\hline \multirow{2}{*}{$\begin{array}{c}101 \\
\text { Desequi- }\end{array}$} & \multirow{3}{*}{1} & \multirow{3}{*}{$110 \%$} & Polar & \multicolumn{3}{|c|}{ Não converge } \\
\hline & & & Retangular & 6,76 & $-1,66$ & $1,04-1,35$ \\
\hline librado & & & Injeção & 6,83 & 0,99 & $1,02-1,07$ \\
\hline \multirow{9}{*}{$\begin{array}{c}120 \\
\text { Equi- } \\
\text { librado }\end{array}$} & \multirow{3}{*}{1} & \multirow{3}{*}{$100 \%$} & Polar & 4,25 & 2,13 & $0,87-1,04$ \\
\hline & & & Retangular & 4,13 & $-2,61$ & $1,04-1,43$ \\
\hline & & & Injeção & 4,13 & $-2,61$ & $1,04-1,43$ \\
\hline & \multirow{3}{*}{2} & \multirow{3}{*}{$100 \%$} & Polar & 4,48 & 2,05 & $0,89-1,04$ \\
\hline & & & Retangular & 4,27 & $-2,44$ & $1,04-1,42$ \\
\hline & & & Injeção & 4,27 & $-2,44$ & $1,04-1,42$ \\
\hline & \multirow{3}{*}{1} & \multirow{3}{*}{$120 \%$} & Polar & 5,02 & $-0,32$ & $1,04-1,23$ \\
\hline & & & Retangular & 5,01 & $-0,69$ & $1,04-1,27$ \\
\hline & & & Injeção & 5,01 & $-0,69$ & $1,04-1,27$ \\
\hline
\end{tabular}

Para sistemas mal-condicionados, equilibrados ou não, observa-se a não convergência do processo iterativo em determinadas situações. Neste contexto, a metodologia polar não converge em alguns dos casos estudados. Por outro lado, os métodos retangulares e injeção de corrente convergem em todos os casos. Além disto, quando as metodologias convergem, nem sempre atingem a mesma solução. Desta forma, para esta situação de mal-condicionamento é difícil a princípio definir a melhor metodologia a ser utilizada na solução do problema de fluxo de potência trifásico.

Na maioria dos casos analisados, as formulações retangulares e injeção de corrente convergem para a mesma solução. Tal situação pode talvez ser explicada pelo fato de que ambas utilizam as coordenadas retangulares da tensão como variáveis de estado. Apesar de usarem equações básicas de potência e de corrente, respectivamente, tais equações estão matematicamente inter-relacionadas, o que corrobora a obtenção da mesma solução.

As formulações retangular convencional e retangular com correção de geração de potência reativa convergem de forma idêntica quando não há a presença de barras PV. Mesmo havendo a presença de tais barras, verifica-se na quase totalidade dos casos simulados, que ambas as formulações convergem no mesmo número de iterações e atingem a mesma solução.

Finalizando, tendo em vista toda a gama de resultados simulados, conclui-se que para a solução geral do problema do fluxo de potência trifásico, todas as metodologias apresenta- das são candidatas em potencial quando os sistemas elétricos em estudo são bem-condicionados. No entanto, tais metodologias devem ser convenientemente testadas e avaliadas frente a sistemas mal-condicionados, sem que haja a princípio, qualquer tipo de preferência sobre uma determinada metodologia. Tal afirmativa vem do fato que a convergência do processo iterativo depende de vários fatores, dentre eles, as condições iniciais, as equações básicas de definição de cada metodologia e, finalmente, de determinadas características inerentes a cada um dos sistemas, que podem em conjunto deteriorar a aplicabilidade de qualquer um dos métodos de solução do fluxo de potência trifásico numa situação específica.

\section{REFERÊNCIAS}

Castro C. A., L. M. C. Braz (1997) Uma nova abordagem para a solução do problema de fluxo de carga pelo método de Newton com otimização do fator de passo. Controle \& Automação, Vol. 8, nº. 3, pp 121-133.

Chen T. e W. Yang (2001) Analysis of multi-grounded fourwire distribution systems considering the neutral grounding. IEEE Transactions on Power Delivery, Vol. 16, $\mathrm{n}^{\circ} .4, \mathrm{pp} 710-717$.

Chen T. H., M. S. Chem, K. J. Hwang, P. Kotas e E. A. Chebi (1991) Distribution system power flow analysis. IEEE Transactions on Power Delivery, Vol. 6, n ${ }^{\circ}$. 3, pp 11461152.

Da Costa, V. M., N. Martins e J. L. R. Pereira (1999) Developments in the Newton Raphson power flow formulation based on current injections. IEEE Transactions on Power Systems, Vol. 14, n ${ }^{o}$. 4, pp 1320-1326.

Das D., H. S. Nagi e D. P. Kothari (1994) Novel method for solving radial distribution networks. Proceedings on Generation, Transmission and Distribution Systems, Vol. 141, $\mathrm{n}^{\circ}$. 4, pp 291-298.

De Oliveira M. L. e V. M. Da Costa (2004) Solução do fluxo de potência de segunda ordem para sistemas de distribuição, Congresso Brasileiro de Automática, Gramado, Brasil.

Expósito A. G. e E. R. Ramos (1999) Reliable load flow technique for radial distribution networks. IEEE Transactions on Power Systems, Vol. 14, n ${ }^{o} .3$, pp 1063-1069.

Ferreira C. A., V. M. Da Costa (2005) A Second Order Power Flow Based on Current Injection Equations. Electrical Power and Energy Systems, vol. 27, pp 254-263.

Garcia A. V. e M. G. T. Zago (1996) Three-phase fast decoupled power flow for distribution networks. Proceedings 
on Generation, Transmission and Distribution Systems, Vol. 143, ${ }^{\circ}$. 2, pp 188-192.

Garcia P. A. N., J. L. R. Pereira, S. Carneiro Jr.,V. M. Da Costa e N. Martins (2000) Three-phase power flow calculations using the current injection method. IEEE Transactions on Power Systems, Vol. 15, $\mathrm{n}^{\circ} .2$, pp 508514.

Garcia P . A. N., J. L. R. Pereira e S. Carneiro Jr. (2001a) Fluxo de potência trifásico por injeção de corrente: parte 1 - formulação básica. Controle \& Automação, Vol.12, ${ }^{\circ} .3$, pp 178-187.

Garcia P. A. N., J. L. R. Pereira e S. Carneiro Jr. (2001b) Jr. (2001b) Fluxo de potência trifásico por injeção de corrente: parte 2 - controles e dispositivos FACTS. Controle \& Automação, Vol.12, ${ }^{\circ}$. 3, pp 188-196

Garcia P. A. N., J. L. R. Pereira, S. Carneiro Jr., M. P. Vinagre e F. V. Gomes (2004) Improvements in representation of PV buses on three-phase distribution power flow. IEEE Transactions on Power Delivery, Vol. 19, $\mathrm{n}^{\circ} .2$, pp 894-896.

Luo G. X. e A. Semlyen (1990) Efficient load flow for large weakly meshed networks. IEEE Transactions on Power Systems, Vol. 5, n ${ }^{\circ}$. 4, pp 1309-1316

Teng J. H. (2003) A direct approach for distribution system load flow solutions. IEEE Transactions on Power Delivery, Vol. 18, $\mathrm{n}^{\circ} .2$, pp 882-887.

Zimmerman R. D. (1995) Fast decoupled power flow for unbalanced radial distribution systems. IEEE Transactions on Power Systems, Vol. 10, $\mathrm{n}^{o}$. 2, pp 2045-2052. 